\title{
Legendre-transform structure derived from quantum theorems
}

\author{
S.P. Flego ${ }^{2}$, A. Plastino ${ }^{1,3}$, and A.R. Plastino ${ }^{3,4}$ \\ ${ }^{1}$ Universidad Nacional de La Plata, \\ Instituto de Física (IFLP-CCT-CONICET), \\ C.C. 727, 1900 La Plata, Argentina and \\ Universitat de les Illes Balears and IFISC-CSIC, 07122 Palma de Mallorca, Spain \\ ${ }^{2}$ Universidad Nacional de La Plata, Facultad de Ingeniería, \\ Depto. de Ciencias Básicas, 1900 La Plata, Argentina \\ 3 CREG-University of La Plata-CONICET \\ ${ }^{4}$ Instituto Carlos I de Fisica Teorica y Computacional and \\ Departamento de Fisica Atomica, Molecular y Nuclear, \\ Universidad de Granada, Granada, Spain
}

\begin{abstract}
By recourse to i) the Hellmann-Feynman theorem and ii) the Virial one, the information-optimizing principle based on Fisher's information measure uncovers a Legendre-transform structure associated with Schrödinger's equation, in close analogy with the structure that lies behind the standard thermodynamical formalism. The present developments provide new evidence for the information theoretical links based on Fisher's measure that exist between Schrödinger's equation, on the one hand, and thermodynamics/thermostatistics on the other one.
\end{abstract}

KEYWORDS: Virial theorem, Hellmann-Feynman theorem, Fisher Information, MaxEnt, Reciprocity relations. 


\section{INTRODUCTION}

The thermodynamical formalism is characterized by its Legendre transform structure [1, 2]. Legendre transformations allow us to express fundamental thermal equations in terms of a set of independent variables chosen to be convenient for a given problem [1, 2]. In a more general context, Legendre transform structures arise naturally in physical theories or models that are based upon entropic or information theoretical optimization principles. An example is that of references [3-6], that purports to rederive on such a basis the principles of statistical mechanics. Here we will explore Schrödinger's non-relativistic equation for links with the Legendre transform framework.

The background for the present considerations was provided by Jaynes, who established in the 50's a perdurable link between Information Theory, Thermodynamics, and Statistical Mechanics [7, 8]. At its core one finds a variational technique involving extremization of Shannon's logarithmic information measure

$$
S=-\sum_{k} p_{k} \ln p_{k}
$$

subject to constraints imposed by the a priori knowledge at hand concerning the system of interest. By identifying Shannon's measure with the thermodynamic entropy a new foundation for statistical mechanics was thereby obtained based on a general statistical inference prescription [7, 8]. The ensuing methodology is usually known as the MaxEnt-one [7, 8]. The MaxEnt approach provides an insightful interpretation of the role played by the Legendre transform referred to above, that is summarized in the Appendix.

An approach similar to the one advanced by Jaynes was successfully developed many years later replacing Shannon's $S$ above by Fisher's information measure (FIM) I [10 13] (see Section III below). Such developments provided an additional perspective within the socalled Wheeler's program of establishing a foundation for the basic laws of physics based on

concepts from information theory [14]. Considerable effort has been expended recently on exploring the physical implications of Fisher information. Indeed, the Fisher Information measure has been successfully applied to the study of several physical scenarios (as a nonexhaustive set, see for instance [12, $15-28]$ ).

Both thermal-information connections, Shannon's and Fisher's, are made by means of a set if first-derivative relations (the Legendre structure) that involve i) the Lagrange multipliers 
that emerge from the variational process, ii) the information quantifier $(S$ or $I)$, and iii) the expectation values that constitute the input, a-priori information on the system of interest. In the Fisher's case a Schrödinger-like equation is involved, a fact of paramount importance for our present purposes.

We will show that the virial and Hellmann-Feynman theorems, essential quantum features, straightforwardly lead to a Legendre-transform structure. After a preliminary presentation in Section II we recapitulates the essential ingredients of Fisher's thermodynamics [21] in Section III. Our main result are derived in Section IV.

\section{PRELIMINARIES}

\section{A. Virial theorem}

For any quantum system in stationary state, with a Hamiltonian does not involve time explicitly,

$$
H=-\frac{\hbar^{2}}{2 m} \vec{\nabla}+U(\vec{x})
$$

the virial theorem states that [29]

$$
\left\langle-\frac{\hbar^{2}}{m} \vec{\nabla}\right\rangle=\langle\vec{x} \cdot \vec{\nabla} U(\vec{x})\rangle
$$

where the expectation value is taken for stationary states of the Hamiltonian.

\section{B. Feynman-Hellmann theorem}

The Feynman-Hellmann theorem (HFT) [30 34] establishes the relationship between perturbations in an operator on a complex inner product space and the corresponding perturbations in the operator's eigenvalues. It shows that to compute the derivative of an eigenvalue with respect to a parameter of the operator we need only know the corresponding eigenvector and the derivative of the operator. More to the point, the Hellmann-Feynman (HF) theorem refers to a parameter dependent eigen-system. It asserts that, in the case of a hermitian op-

erator $H(\lambda)$ (whose eigenvectors are $\psi_{i}$ ), a non-degenerate eigenvalue $E_{i}$ varies with respect to the parameter $\lambda$ according to the expression 


$$
\frac{\partial E_{i}}{\partial \lambda}=\left\langle\psi_{i}\left|\frac{\partial H}{\partial \lambda}\right| \psi_{i}\right\rangle
$$

The theorem has a rich history and many applications, that can be consulted in [34]. The FH theorem can be proved to hold for exact eigenstates and also for variationally determined states [30].

\section{THE FISHER THERMAL FORMALISM}

\section{A. Basics results}

This formalism was advanced in Ref. [13]. One considers a system that is specified by a physical parameter $\theta$, while the quantity $f(\theta)$ determines the normalized probability distribution function (PDF) for it. Fisher's Information Measure (FIM) I gets defined as

$$
I=\int d x f(x, \theta)\left\{\frac{\partial}{\partial \theta} \ln [f(x, \theta)]\right\}^{2} .
$$

Fix attention upon translational families, which are mono-parametric distribution ones of the form

$$
f(x, \theta)=f(x-\theta)
$$

known up to the shift parameter $\theta$. All members of the family possess identical shape, and for them FIM adopts the simpler form

$$
I=\int d x f(x)\left\{\frac{\partial}{\partial x} \ln [f(x)]\right\}^{2} .
$$

We are interested in a system that is specified by a set of $M$ physical parameters $\mu_{k}$. More to the point

$$
\mu_{k}=\left\langle A_{k}\right\rangle, \quad \text { with } \quad A_{k}=A_{k}(x) \quad(k=1, \ldots, M) .
$$

The set of $\mu_{k}$-values represents the empirical information at hand. If the pertinent probability distribution function (PDF) is $f(x)$, then

$$
\left\langle A_{k}\right\rangle=\int d x A_{k}(x) f(x), \quad k=1, \ldots, M
$$


These mean values will play the role of extensive thermodynamical variables [13]. The relevant $\operatorname{PDF} f(x)$ for us is the one that extremizes (5) subject to i) the prior conditions (6) and, of course, ii) the normalization condition

$$
\int d x f(x)=1
$$

Accordingly, the extremization problem that we face is

$$
\delta\left(I-\alpha \int d x f(x)-\sum_{k=1}^{M} \lambda_{k} \int d x A_{k}(x) f(x)\right)=0
$$

where we have $(M+1)$ Lagrange multiplier. Variation leads to

$$
\left[\frac{1}{f^{2}}\left(\frac{\partial f}{\partial x}\right)^{2}+\frac{\partial}{\partial x}\left(\frac{2}{f} \frac{\partial f}{\partial x}\right)\right]+\alpha+\sum_{k=1}^{M} \lambda_{k} A_{k}(x)=0
$$

It is convenient [13, 20, 36] to introduce a function $\psi(x)$ via the identification $\psi(x)^{2}=f(x)$ so that Eq. (9) acquires a wave equation form (SWE)

$$
-\frac{1}{2} \frac{\partial^{2}}{\partial x^{2}} \psi-\sum_{k=1}^{M} \frac{\lambda_{k}}{8} A_{k} \psi=\frac{\alpha}{8} \psi
$$

which can be formally interpreted as a Schrödinger equation for a particle of unit mass moving in the effective, "information" pseudo-potential [Cf. Eq. (6)]

$$
U=U(x)=-\frac{1}{8} \sum_{k=1}^{M} \lambda_{k} A_{k}(x) .
$$

The Lagrange multiplier $(\alpha / 8)$ plays the role of an energy eigenvalue $E=\alpha / 8$. The Lagrange parameters $\lambda_{k}$ are fixed, of course, by recourse to the input prior information. Notice that the eigen-energies $\alpha / 8$ yield automatically the value of the Lagrange multiplier associated to normalization. The square of the solutions $\psi$ is the desired PDF

$$
\psi(x)^{2}=f(x)
$$

\section{B. Finding a convenient way of using FIM}

It is now important to establish a new form of expressing Fisher's information measure as a function of $\psi$. One substitutes (12) into Eq. (15) to find 


$$
I=\int d x f\left(\frac{\partial \ln f}{\partial x}\right)^{2}=\int d x \psi_{n}^{2}\left(\frac{\partial \ln \psi_{n}^{2}}{\partial x}\right)^{2}=4 \int d x\left(\frac{\partial \psi_{n}}{\partial x}\right)^{2}
$$

which can be re-expressed as

$$
I=-4 \int \psi_{n} \frac{\partial^{2}}{\partial x^{2}} \psi_{n} d x=-4\left\langle\frac{\partial^{2}}{\partial x^{2}}\right\rangle
$$

Now, using the SWE (10) one obtains

$$
I=\int \psi_{n}\left(\alpha+\sum_{k=1}^{M} \lambda_{k} A_{k}\right) \psi_{n} d x
$$

Finally, the prior conditions ([6]) and the normalization condition (7) allow one to express $I$ in the quite convenient fashion

$$
I\left(\left\langle A_{1}\right\rangle, \ldots,\left\langle A_{M}\right\rangle\right)=\alpha+\sum_{k=1}^{M} \lambda_{k}\left\langle A_{k}\right\rangle
$$

\section{Fisher thermodynamics}

The connection between the solutions of Eq. (10) and thermodynamics has been established in Refs. [13] and [15]. We summarize now the main details. The reciprocity relations (41) and their Fisher-counterparts are an expression of the Legendre-transform structure of thermodynamics [9, 21] and constitute its essential formal ingredient [1]. It is of the essence that they also hold for the Fisher treatment. Standard thermodynamic makes use of the

derivatives of the entropy $S$ with respect to both the $\lambda_{i}$ and $\left\langle A_{i}\right\rangle$ quantities (for instance, pressure and volume, respectively).

Analogous properties of $\partial I / \partial \lambda_{i}$ and $\partial I / \partial\left\langle A_{i}\right\rangle$ are valid as well [13]. We start with (16) and consider its Legendre transform, that we call $\alpha$, i.e.,

$$
\alpha\left(\lambda_{1}, \ldots, \lambda_{M}\right)=I\left(\left\langle A_{1}\right\rangle, \ldots,\left\langle A_{M}\right\rangle\right)-\sum_{k=1}^{M} \lambda_{k}\left\langle A_{k}\right\rangle
$$

so that

$$
\frac{\partial \alpha}{\partial \lambda_{i}}=-\left\langle A_{i}\right\rangle
$$


and recall two expressions obtained in [13], namely,

$$
\frac{\partial I}{\partial\left\langle A_{k}\right\rangle}=\lambda_{k}
$$

and

$$
\frac{\partial I}{\partial \lambda_{i}}=\sum_{k}^{M} \lambda_{k} \frac{\partial\left\langle A_{k}\right\rangle}{\partial \lambda_{i}}
$$

which is a generalized Fisher-Euler theorem. It is instructive to glance at the Appendix at this point to note that entirely similar relations are obeyed by the ordinary Gibbs-Boltzmann entropy $S$. On the basis of such an observation, it seems natural to consider that the three reciprocity relations above should allow one to speak of a "Fisher-thermodynamics" [15]. Curiously enough, it can be shown that the HF theorem can be looked at as a reciprocity relation of the type (18) [35].

\section{A QUANTAL-FISHER CONNECTION}

We begin here to develop the original contents of this presentation. Let us consider now that Eq. (10) is an ordinary Schrödinger wave equation for a particle of unit mass in which the Lagrange multiplier $(\alpha / 8)$ plays the role of an energy eigenvalue $E=\alpha / 8$. Remark that $U(x)$ is taken now to be an actual, physical potential, not an effective, "information" one. This is the starting point.

We emphasize now the fact that our FIM $I$ is now seen to be proportional to the expectation value of the Laplace operator, namely,

$$
I=\int d x f\left(\frac{\partial \ln f}{\partial x}\right)^{2}=-4 \int \psi_{n} \frac{\partial^{2}}{\partial x^{2}} \psi_{n} d x=-4\left\langle\frac{\partial^{2}}{\partial x^{2}}\right\rangle
$$

where $\psi_{n}$ are the eigenfunctions of

$$
\left[-\frac{1}{2} \frac{\partial^{2}}{\partial x^{2}}-\frac{1}{8} \sum_{k} \lambda_{k} A_{k}\right] \psi=\frac{1}{8} \alpha \psi
$$

We take it for granted that, as customary, the potential $U$ admits of a series-expansion (powers of $x^{k}$ ) of the form 


$$
U(x)=-\frac{1}{8} \sum_{k} \lambda_{k} A_{k} \equiv-\frac{1}{8} \sum_{k} \lambda_{k} x^{k}=\sum_{k} a_{k} x^{k} ; \quad-\lambda_{k} / 8=a_{k} .
$$

Thus, the $A_{k}$ in the preceding Sections become here $x^{k}$-moments and one assumes that the expansion is good enough if $M$ terms of them are included. The $\lambda_{k}$ are now the expansioncoefficients and not Lagrange multipliers. A Fisher's measure is to be constructed with these coefficients. Recourse to the Virial theorem (2) allows us to cast the FIM-expression (21) in the fashion

$$
I=-\sum_{k=1}^{M} \frac{k}{2} \lambda_{k}\left\langle A_{k}\right\rangle
$$

Now, replacing (24) into (16) and solving for $\alpha$ one finds

$$
\alpha=-\sum_{k=1}^{M}\left(\frac{k}{2}+1\right) \lambda_{k}\left\langle A_{k}\right\rangle
$$

having thus obtained two expressions that pave a direct road towards our present goal.

\section{A. Hellmann-Feynman and Virial theorems imply reciprocity relations}

In this subsection we are going to show that Eqs. (24), (25), and the Hellmann-Feynman theorem (3) jointly lead to Fisher-reciprocity relations. It being clear up this point that the $\lambda$ 's are expansion-coefficients, we will speak herefrom only " $\lambda$-language".

In one dimensional scenarios, the eigenfunctions $\psi(x)$ of (10) are real. We appeal now to the Hellmann-Feynman theorem and obtain

$$
\frac{\partial}{\partial \lambda_{k}}\left(\frac{\alpha}{8}\right)=\left\langle\psi\left|\frac{\partial H}{\partial \lambda_{k}}\right| \psi\right\rangle=\left\langle\psi\left|-\frac{1}{8} A_{k}\right| \psi\right\rangle \quad \longrightarrow \quad \frac{\partial \alpha}{\partial \lambda_{k}}=-\left\langle A_{k}\right\rangle,
$$

thus discovering that the HF theorem immediately implies the reciprocity relation (18).

It is clear that differentiating (25) with respect to $\lambda_{n}$ yields

$$
\frac{\partial \alpha}{\partial \lambda_{n}}=-\left(\frac{n}{2}+1\right)\left\langle A_{n}\right\rangle-\sum_{k=1}^{M}\left(\frac{k}{2}+1\right) \lambda_{k} \frac{\partial\left\langle A_{k}\right\rangle}{\partial \lambda_{n}}
$$

The two relations (26) and (27) result now in 


$$
\frac{n}{2}\left\langle A_{n}\right\rangle=-\sum_{k=1}^{M}\left(\frac{k}{2}+1\right) \lambda_{k} \frac{\partial\left\langle A_{k}\right\rangle}{\partial \lambda_{n}}
$$

We go back to (24) at this point and differentiate it with respect to $\lambda_{n}$ to arrive at

$$
\frac{\partial I}{\partial \lambda_{n}}=-\frac{n}{2}\left\langle A_{n}\right\rangle-\sum_{k=1}^{M} \frac{k}{2} \lambda_{k} \frac{\partial\left\langle A_{k}\right\rangle}{\partial \lambda_{n}} .
$$

At this stage, recourse to the relation (28) allows one to recover the Euler relations

$$
\frac{\partial I}{\partial \lambda_{n}}=\sum_{k=1}^{M} \lambda_{k} \frac{\partial\left\langle A_{k}\right\rangle}{\partial \lambda_{n}}
$$

We also have

$$
\frac{\partial I\left(<A_{1}>, \ldots,<A_{M}>\right)}{\partial \lambda_{n}}=\sum_{k=1}^{M} \frac{\partial I}{\partial\left\langle A_{k}\right\rangle} \frac{\partial\left\langle A_{k}\right\rangle}{\partial \lambda_{n}}
$$

so that, comparing (30) and (31) we immediately obtain

$$
\frac{\partial I}{\partial\left\langle A_{n}\right\rangle}=\lambda_{n}
$$

The three expressions (26) , (30) and (32), obtained by application of the Hellmann-Feynman theorem and the Virial one to Fisher's information measure, are reciprocity relations that in turn constitute a manifestation of an underlying Legendre-invariant structure, analogous to that of thermodynamics, our main result here.

\section{CONCLUSIONS}

In this work we have shown that, if Fisher's measure $I$ is associated to a Schrödinger wave equation (SWE), as it happens whenever one extremizes it subject to appropriate constraints, two theorems intimately linked to the SWE, the Hellmann-Feynman and Virial ones, automatically lead to Jaynes-like reciprocity relations involving the coefficients of the seriesexpansion of the potential function. One may then dare to assert that a Legendre-transform 
structure seems to underly the one-dimensional non-relativistic Schrödinger's equation, a rather surprising finding.

Acknowledgments- This work was partially supported by the Project FQM-2445 of the Junta de Andalucia, Spain. 


\section{APPENDIX: MAXENT AND LEGENDRE STRUCTURE}

The classical MaxEnt probability distribution function (PDF), associated to BoltzmannGibbs-Shannon's logarithmic entropy, is given by [7, 8]

$$
f(\text { MaxEnt })=f(x)=\exp \left\{-\left[\Omega+\sum_{i=1}^{M} \lambda_{i} A_{i}(x)\right]\right\}
$$

with [7, 8]

$$
\begin{gathered}
\Omega\left(\lambda_{1}, \ldots, \lambda_{M}\right)=\ln \left\{\int d x\left[\exp \left(-\sum_{i=1}^{M} \lambda_{i} A_{i}(x)\right)\right]\right\} \equiv-\lambda_{o}, \\
\frac{\partial \Omega\left(\lambda_{1}, \ldots, \lambda_{M}\right)}{\partial \lambda_{j}}=-\left\langle A_{j}\right\rangle, \quad(j=1, \ldots, M),
\end{gathered}
$$

and

$$
S=\Omega+\sum_{i=1}^{M} \lambda_{i}\left\langle A_{i}\right\rangle
$$

entailing

The Euler theorem holds [8]

$$
d S=\sum_{i=1}^{M} \lambda_{i} d\left\langle A_{i}\right\rangle
$$

$$
\frac{\partial S}{\partial \lambda_{i}}=\sum_{k} \lambda_{k} \frac{\partial\left\langle A_{k}\right\rangle}{\partial \lambda_{i}}
$$

and, using (36), one arrives to

$$
\begin{aligned}
d S & =\sum_{i=1}^{M} \lambda_{i} d\left\langle A_{i}\right\rangle \Longrightarrow \frac{\partial S}{\partial\left\langle A_{i}\right\rangle}=\lambda_{i} \\
S & =S\left(\left\langle A_{1}\right\rangle, \ldots,\left\langle A_{M}\right\rangle\right) .
\end{aligned}
$$

Effecting now the Legendre transform

$$
\Omega=\Omega\left(\lambda_{1}, \ldots, \lambda_{M}\right)=S-\sum_{i=1}^{M} \lambda_{i}\left\langle A_{i}\right\rangle,
$$

one immediately ascertains that reciprocity holds, namely,

$$
\frac{\partial S}{\partial\left\langle A_{j}\right\rangle}=\lambda_{j} \quad \text { and } \quad \frac{\partial \Omega}{\partial \lambda_{j}}=-\left\langle A_{j}\right\rangle ; j=1, \ldots, M
$$


where the second set of equations, together with (34), yield the Lagrange multipliers as a function of the input information regarding expectation values [8]. The reciprocity relations (38) + (41) are a manifestation of the Legendre-transform structure of thermodynamics [1, 9] and its most salient structural mathematical feature.

[1] Deslog E. A., Thermal Physics (Holt, Rinehart and Winston, New York, 1968).

[2] H. B. Callen, Thermodynamics (Wiley, NY, 1960).

[3] E. Curado, A. Plastino, Phys. Rev. E 72 (2005) 047103.

[4] A. Plastino, E. Curado, Physica A 365 (2006) 24.

[5] A. Plastino, E. Curado, Physica A 386 (2007) 155.

[6] E. Curado, F. Nobre, A. Plastino, Physica A 389 (2010) 970.

[7] Jaynes E.T., Information Theory and Statistical Mechanics, Phys. Rev. 1957 106, 620-630.

[8] Katz, A. Principles of Statistical Mechanics: The Information Theory Approach (Freeman and Co.: San Francisco, 1967).

[9] A. Plastino, A. R. Plastino, Phys. Lett. A 226 (1997) 257.

[10] B. R. Frieden, Phys. Rev. A 41 (1990) 4265.

[11] B. R. Frieden, Physics from Fisher information measure (Cambridge, University Press; Cambridge, 1998).

[12] B. R. Frieden, B. H. Soffer, Phys. Rev. E 521995 (1995) 2274.

[13] B. R. Frieden, A. Plastino, A. R. Plastino, B. H. Soffer, Phys. Rev. E 601999 (1999) 48.

[14] Wheeler J.A., Complexity, entropy and the physics of information; (W.H. Zurek Ed.; Addison Wesley; New York, 1991, pp. 3-28).

[15] S. P. Flego, B. R. Frieden, A. Plastino, A. R. Plastino, B. H. Soffer, Phys. Revi. E 68 (2003) 016105.

[16] A. R. Plastino, A. Plastino, Phys. Rev. E 54 (1996) 4423.

[17] A. Plastino, A. R. Plastino, H. G. Miller, Phys. Lett. A 235 (1997) 129.

[18] A. R. Plastino, M. casas, A. Plastino, Phys. Lett. A 246 (1998) 498.

[19] F. Olivares, F. Pennini, A. Plastino, Physica A 389 (2010) 2218.

[20] Silver R. N. in E. T. Jaynes: Physics and Probability (W. T. Grandy and P. W. Milonni, Eds.; Cambridge University Press; Cambridge, 1992). 
[21] F. Pennini, A. Plastino, Phys. Rev. E 71 (2005) 047102.

[22] V. Kapsa, L. Skala and J. Chen, Physica E 42 (2010) 293.

[23] B.R. Frieden and B.H. Soffer, Physica A 388 (2009) 1315.

[24] M. R. Ubriaco. Phys. Lett. A 373 (2009) 4017.

[25] S. Lopez-Rosa, J.C. Angulo, J.S. Dehesa and R.J. Yanez, Physica A 387 (2008) 2243.

[26] K.D. Sen, J. Antolin and J.C. Angulo, Phys. Rev. A 76 (2007) 032502.

[27] A. Nagy, Chem. Phys. Lett. 449 (2007) 212.

[28] A. Nagy, Chem. Phys. Lett. 425 (2006) 154.

[29] W. Geiner and B. Müller, Quantum mechanics. An Introduction(Springer, Berlin, 1988).

[30] W. Namgung, Journal of the Korean Physical Society, 32 (1998) 647.

[31] H. G. A. Hellmann, Z. Phys, 85 (1933) 180.

[32] R. P. Feynman, Phys. Rev. 56 (1939) 340.

[33] D. J. Griffiths, Introduction to Quantum Mechanics (Prentice Hall, Englewood Cliffs, NJ, 1995).

[34] David W- Wallace, An introduction to Hellmann-Feynman theory(Master Thesis, University of Central Florida, Orlando, Florida, 2005 (unpublished)).

[35] S. P. Flego, A. Plastino, A. R. Plastino, unpublished.

[36] Richards P. I., Manual of mathematical physics (Pergamon Press; London, 1959). 